02

\title{
Искажения в ИК спектрах, связанные с условиями регистрации. II. Влияние рассеяния
}

\author{
(C) Р.Г. Новиков ${ }^{1}$, Р. Конопницкий ${ }^{2}$, А.А. Цыганенко ${ }^{1, ~}$ \\ ${ }^{1}$ Санкт-Петербургский государственный университет, \\ 199034 Санкт-Петербург, Россия \\ ${ }^{2}$ École nationale supérieure de physique de Strasbourg, \\ Illkirch-Graffenstaden, France \\ ฯ e-mail: atsyg@ya.ru
}

Поступила в редакцию 16.12.2017 г.

В окончательной редакции 05.01.2018 г.

\begin{abstract}
Рассмотрены искажения формы полос поглощения сильно рассеивающих образцов в спектрах молекул, адсорбированных на поверхности дисперсных твердых тел. Показано, что влияние рассеяния на ИК спектры дисперсных образцов не ограничивается ослаблением интенсивности проходящего света, а влияет на контур спектральных полос вследствие изменений показателя преломления вещества в области полос поглощения. На примере диоксида углерода, адсорбированного на цеолите NaX, продемонстрировано два метода учета вклада рассеяния в спектры поверхностных соединений: регистрация спектра „диффузного пропускания“ и погружение образца в иммерсионную жидкость, в качестве которой использован жидкий кислород. Полученные спектры свидетельствуют, что при насыщении цеолита адсорбатом полоса антисимметричного валентного колебания молекул $\mathrm{CO}_{2}$ уширяется и обнаруживает сложную структуру в результате резонансного диполь-дипольного взаимодействия между адсорбированными молекулами.
\end{abstract}

DOI: $10.21883 /$ OS.2018.05.45942.294-17

\section{Введение}

Количественный спектральный анализ основывается на использовании закона Ламберта-Беера, который обычно применяется без учета эффектов рассеяния и отражения света на границах раздела. Однако оба эти эффекта вносят вклад в ослабление проходящего света, который в определенных условиях может оказаться сравнимым с поглощением света исследуемым образцом. При изучении спектров пропускания уменьшение количества света, пришедшего на детектор вне зависимости от причины, отображается как поглощение. Вследствие сильной зависимости показателя преломления от частоты вблизи полос поглощения как отражение, так и рассеяние могут приводить к заметным искажениям контура спектральных полос в наблюдаемых спектрах. Влияние отражения света на границах раздела между исследуемым веществом и окнами кюветы или подложкой и на ИК спектры поглощения пленок, жидкостей и адсорбированных молекул было рассмотрено нами ранее [1].

В настоящей работе мы исследовали искажения, вызванные рассеянием, в спектрах дисперсных твердых тел на примере цеолита $\mathrm{NaX}$ с адсорбированным диоксидом углерода. Рассеяние может вносить существенный вклад в спектры сильно рассеивающих образцов, в частности влияя на контуры полос адсорбированных на их поверхности сильно поглощающих молекул. Как было недавно показано, контур наиболее интенсивных полос колебания $v_{3}$ таких молекул, как $\mathrm{SF}_{6}$ [2] или $\mathrm{CF}_{4}$ [3], при насыщающих покрытиях несет информацию о геометрии поверхности, обнаруживая характерное расщепление, неодинаковое для плоской поверхности оксида или цепочек молекул, внедренных в каналы цеолитов. Частоты упомянутого колебания $v_{3}$ этих молекул лежат в области 1300-900 $\mathrm{cm}^{-1}$, где потери света на рассеяние пренебрежимо малы, и оно слабо влияет на форму контура полос.

Молекула $\mathrm{CO}_{2}$, широко используемая как зонд для количественной характеризации как основных, так и кислотных свойств центров поверхности цеолитов и оксидов методом ИК спектроскопии [4-6], имеет наиболее интенсивную полосу поглощения антисимметричного валентного колебания $v_{2}$ при $2349 \mathrm{~cm}^{-1}$ [7]. Интегральный коэффициент поглощения этой полосы столь велик $\left(\varepsilon=320 \mathrm{~cm}^{2} /\right.$ molecule $\left.\times \mathrm{m}[8]\right)$, что в случае цеолитов количественные оценки покрытия удобнее делать по интенсивности полос поглощения составных колебаний, например по полосе колебания $v_{1}+v_{2}$ при частоте около $3714 \mathrm{~cm}^{-1}$ [6].

Для записи контура полосы $v_{2}$ с разумным значением поглощения в максимуме в условиях насыщения требуется изготовление образцов „толщиной“ менее $5 \mathrm{mg} / \mathrm{cm}^{2}$. Изготовить столь тонкие образцы цеолита с использованием обычной методики прессования не представляется возможным, и с этой целью мы применили методику помещения тонкого слоя цеолита между пластинками из материала, прозрачного для ИК излучения: флюорита или кремния. Такие образцы отличаются более сильным рассеянием по срав- 
нению с прессованными, и их использование затруднено необходимостью выжидать, пока молекулы адсорбируемого газа продиффундируют в центральную часть образца, но интенсивность полос как объемного поглощения цеолита, так и адсорбированных на нем молекул не превосходит 2.5, что обеспечивает низкий уровень шумов и воспроизводимость получаемых спектров.

Для учета искажений контура полосы колебания $v_{2}$ в ИК спектрах адсорбированного $\mathrm{CO}_{2}$ нами были использованы два разных метода. Первый заключался в регистрация спектров „диффузного пропускания“ [9], т.е. спектра света, изменившего направление при прохождение через образец вследствие рассеяния. Второй способ состоял в пропитке образца иммерсионной жидкостью, в качестве которой мы использовали жидкий кислород при $77 \mathrm{~K}$.

\section{Методика эксперимента}

Для регистрации спектров термовакуумной обработки образцов и адсорбции $\mathrm{CO}_{2}$ использовалась ранее описанная [10] низкотемпературная вакуумная кювета из нержавеющей стали с двумя парами окон и кварцевой трубкой для тренировки образцов. Охлаждаемые внутренние окна из $\mathrm{ZnSe}$, уплотненные прокладками из индия, позволяли регистрировать спектр в ИК диапазоне до $500 \mathrm{~cm}^{-1}$. Для обеспечения теплового контакта образца с холодными стенками кюветы при регистрации спектров во внутренний объем кюветы напускалось около 0.5 Torr гелия. Давление внутри кюветы измеряли с помощью датчика фирмы Edwards диапазоном 12 Torr и точностью $\pm 10^{-3}$ Torr. Для конденсации кислорода во внутреннем объеме кюветы с образцом дополнительное охлаждение кюветы ниже $77 \mathrm{~K}$ достигалось откачкой испаряющегося азота из объема для хладагента.

Порцию цеолита весом 3-5 mg распределяли ровным слоем по площади около $1 \mathrm{~cm}^{2}$ и зажимали между тонкими пластинками из кремния или флюорита. Перед работой образец медленно вакуумировали при плавном повышении температуры до $673 \mathrm{~K}$ в течение $1 \mathrm{~h}$ и выдерживали в таких условиях около $30 \mathrm{~min}$.

Для адсорбции диоксид углерода в избытке напускался в охлажденный до $77 \mathrm{~K}$ внутренний объем кюветы, после чего температура повышалась до $\sim 140 \mathrm{~K}$, давление - до нескольких Torr, что свидетельствовало о появлении $\mathrm{CO}_{2}$ в газовой фазе. Контроль за покрытием поверхности производился спектрально по росту полосы адсорбированных молекул. После регистрации спектра при насыщающем покрытии часть адсорбата удаляли, откачивая часть газа после повышения температуры образца, после чего вновь охлаждали кювету и записывали спектр.

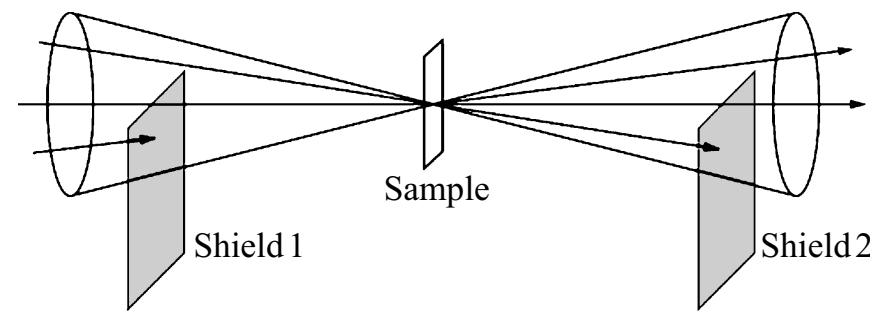

Рис. 1. Схема регистрации спектра „диффузного пропускания“ путем выделения излучения, отклоненного образцом.

Спектры регистрировали на фурье-спектрометре Nicolet 510 с разрешением $4 \mathrm{~cm}^{-1}$. Для обработки спектров использовалась программа OMNIC.

Способ получения спектров „диффузного пропускания“ был описан нами ранее [10]. Для этого в пучок ИК радиации на входе и выходе кюветного отсека вводились две шторки таким образом, что в отсутствие образца интенсивность проходящего света была достаточно малой (рис. 1). Тогда при введении образца на детектор фурьеспектрометра попадает только излучение, отклоненное из-за рассеяния. Интенсивность регистрируемого сигнала при этом оказывается весьма низкой (менее 1\%), однако спектр качественно отличается от обычного. В частности, в нем отсутствует обусловленный рассеянием склон с уменьшением пропускания при повышении частоты.

Для изучения спектров образца, погруженного в жидкий кислород, как и ранее $[11]$, в объем с образцом подавался технический кислород под давлением 200-300 Torr, необходимым для его конденсации при $77 \mathrm{~K}$. Появление полосы индуцированного поглощения кислорода при $1555 \mathrm{~cm}^{-1}$ свидетельствовало о заполнении пространства между окнами жидким кислородом. Однако существенное увеличение прозрачности образца в результате его пропитки жидким кислородом наступало раньше, как только уровень жидкости достигал нижней части образца, и его спектр при дальнейшем повышении уровня практически не менялся.

Использовался коммерческий цеолит $\mathrm{NaX}$ с размером частиц $2-7 \mu \mathrm{m}$. Технический диоксид углерода перед адсорбцией очищали откачкой при вымораживании жидким азотом. Кислород перед впуском в кювету для очистки пропускали через ловушку, охлаждаемую жидким кислородом.

\section{Результаты экспериментов}

ИК спектры $\mathrm{CO}_{2}$, адсорбированного в условиях, близких к насыщению, на тонком образце цеолита $\mathrm{NaX}$, показаны на рис 2. Исходный спектр цеолита, записанный традиционным способом „на пропускание“ (кривая 1), характеризуется сильным ростом поглощения с повышением частоты, а вместо полосы поглощения $\mathrm{CO}_{2}$ при 


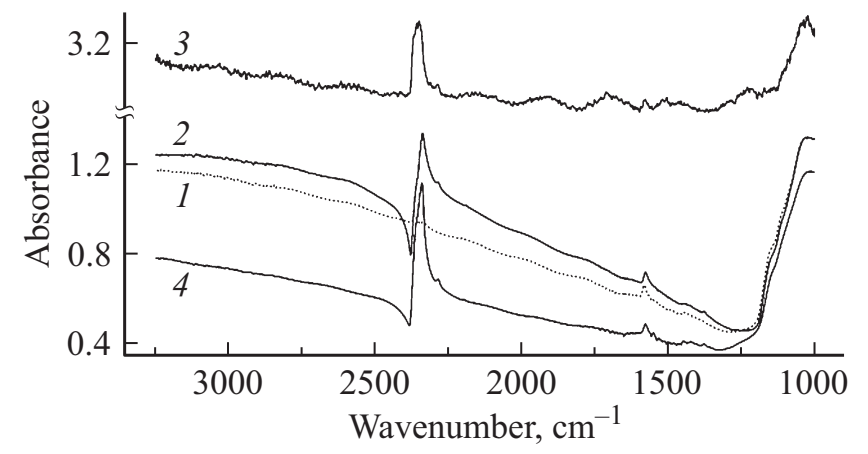

Рис. 2. ИК спектр тонкого слоя цеолита $\mathrm{NaX}$, помещенного между двумя пластинками из кремния, после термовакуумной обработки при $673 \mathrm{~K}(1)$; адсорбции $\mathrm{CO}_{2}$, охлаждения до $77 \mathrm{~K}$ и регистрации в обычном режиме „напросвет“ (2) и в режиме „диффузного пропускания“ (3); после погружения образца в жидкий кислород (4).

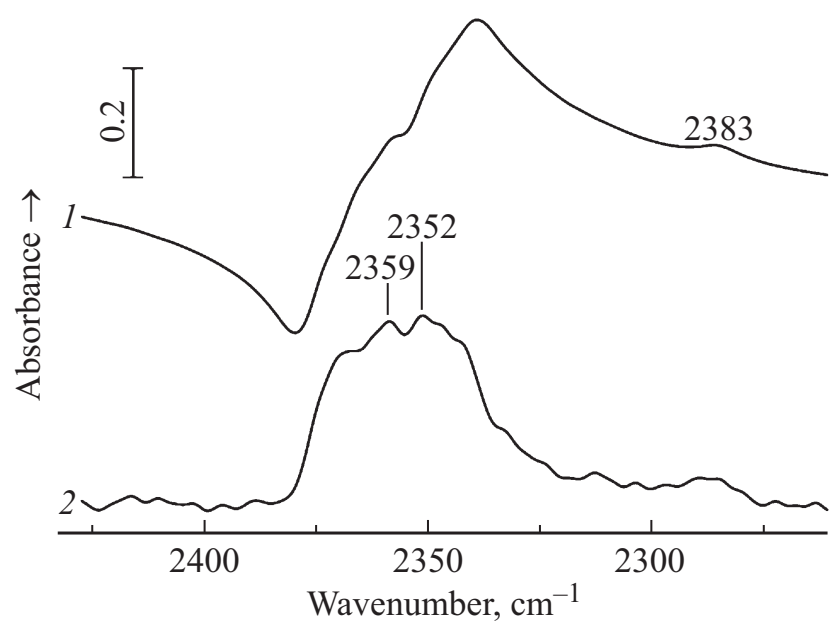

Рис. 3. ИК спектр $\mathrm{CO}_{2}$, адсорбированного на тонком слое цеолита $\mathrm{NaX}$ в условиях, близких к насыщению (1), и спектр „диффузного пропускания“ того же образца (2). Спектры соответствуют кривым 2 и 3 на рис. 2.

$235 \mathrm{~cm}^{-1}$ наблюдается некое подобие дисперсионной кривой, где с высокочастотной стороны от пика поглощения с максимумом при $2347 \mathrm{~cm}^{-1}$ возникает область с пропусканием, увеличенным по отношению к тому же образцу до адсорбции.

Полоса $\mathrm{CO}_{2}$ в спектре 3 , записанном в режиме „диффузного пропускания“", гораздо менее искажена, однако из-за слабого сигнала спектр отличается высоким уровнем шумов. В спектре образца, погруженного в жидкий кислород (кривая 4), напротив, уровень сигнала заметно выше исходного, и в области $2000 \mathrm{~cm}^{-1}$ соответствует уменьшению потерь света в единицах поглощения (absorbance) на 0.5-1.0 в зависимости от толщины образца.

Более детально контур полосы поглощения $\mathrm{CO}_{2}$ показан на рис. 3. В спектре „диффузного пропускания“ (кривая 2) полоса уширена и обнаруживает структуру со слабо выраженными максимумами при 2359 и $2352 \mathrm{~cm}^{-1}$. Тем не менее высокий уровень шумов при низкой интенсивности не позволяет с уверенностью судить о форме контура этой полосы.

Увеличение прозрачности образца при погружении его в жидкий кислород позволяет получить более надежные данные о форме полосы. На рис. 4 спектры 1 и 2 соответствуют кривым 2 и 4 из рис. 2 и показывают изменение спектра адсорбированного $\mathrm{CO}_{2}$ при погружении образца в жидкий кислород. Оба спектра представляют собой сумму потерь энергии излучения на поглощение и рассеяние, но доля рассеяния в спектре до погружения существенно выше. Это означает, что вычитая первый спектр из второго с фактором, подобранным таким образом, чтобы крылья полосы со стороны высоких и низких частот выглядели симметрично, можно надеяться получить в чистом виде спектр поглощения. В полученном таким способом разностном спектре (кривая 3) наблюдается широкая полоса со сложной структурой из нескольких максимумов от 2378 до $2332 \mathrm{~cm}^{-1}$. Примечательно, что ширина полосы на половине высоты достигает почти $60 \mathrm{~cm}^{-1}$, в то время как слабый ее сателлит при $2283 \mathrm{~cm}^{-1}$, очевидно, принадлежащий молекулам изотопомера ${ }^{13} \mathrm{CO}_{2}$, записывается с полушириной не более $10 \mathrm{~cm}^{-1}$. После удаления большей части адсорбата при повышении температуры до 200 К в спектре остается одна полоса с максимумом при $2355 \mathrm{~cm}^{-1}$ (кривая 4), по полуширине не отличающаяся от полосы изотопозамещенных молекул.

\section{Обсуждение результатов}

Приведенные результаты о спектре молекулярно адсорбированного $\mathrm{CO}_{2}$ при умеренном заполнении поверхности находятся в согласии с ранее опубликованными данными. Так, в работе [4] сообщается о полосе $2355 \mathrm{~cm}^{-1}$ и сопутствующей ей слабой полосе изотопного аналога при $2287 \mathrm{~cm}^{-1}$. Это в пределах ошибки эксперимента соответствует спектру 4 на рис. 4 . Согласно [5], молекулярной адсорбции $\mathrm{CO}_{2}$ на атоме натрия в цеолите ZSM-5 соответствуют полосы $2356 \mathrm{~cm}^{-1}$ и слабая $2290 \mathrm{~cm}^{-1}$. Следует отметить, что наблюдаемый изотопный сдвиг при переходе от ${ }^{13} \mathrm{CO}_{2} \mathrm{~K}{ }^{12} \mathrm{CO}_{2}$ в пределах точности эксперимента соответствует вычисленному из приведенных масс фактору 1.0292.

Наблюдавшейся нами полосе ${ }^{13} \mathrm{CO}_{2}$ при $2285 \mathrm{~cm}^{-1}$ должна соответствовать полоса ${ }^{12} \mathrm{CO}_{2}$ при $2352 \mathrm{~cm}^{-1}$. В пределах ошибки эксперимента это совпадает со значением $2355 \mathrm{~cm}^{-1}$ для полосы, наблюдавшейся нами при малых заполнениях, и несколько хуже согласуется с результатами работы [10], где из спектров ,диффузного пропускания“ была выделена одиночная полоса при $2360 \mathrm{~cm}^{-1}$ (при полосе изотопной ${ }^{13} \mathrm{CO}_{2} 2286 \mathrm{~cm}^{-1}$ ).

Совсем по-другому обстоит со спектрами при высоких заполнениях. Здесь как спектры, записанные в режиме 
„диффузного пропускания“, так и полученные путем погружения в жидкий кислород свидетельствуют, что относительно узкой полосе колебания $v_{2}$ примесного изотопомера ${ }^{13} \mathrm{CO}_{2}$ с полушириной не более $12 \mathrm{~cm}^{-1}$ соответствует в 3-5 раз более широкая полоса, имеющая структуру из нескольких плохо разрешенных максимумов. Различие в ширине этой полосы на рис. 3 и 4, а также по сравнению с ранее полученной из спектра „диффузного пропускания“ полосой $2360 \mathrm{~cm}^{-1}$ [2] естественно объяснить разной степенью заполнения поверхности диоксидом углерода в различных экспериментах.

Подобное наблюдавшемуся в этой работе различие спектров преобладающего и примесного изотопа характерно для систем с проявлениями сильного резонансного диполь-дипольного (РДД) взаимодействия. Ранее влияние РДД взаимодействия на контур полос сильно поглощающих молекул было изучено нами в спектрах адсорбированных $\mathrm{SF}_{6}$ [2], $\mathrm{CF}_{4}$ [3] и $\mathrm{NF}_{3}$ [12]. $\mathrm{B}$ результате такого взаимодействия, называемого также динамическим (или в англоязычной литературе dipole coupling), колебания отдельных молекул перестают быть локализованными и превращаются в коллективные колебания ансамбля взаимодействующих частиц подобно тому, как это происходит в молекулярных кристаллах.

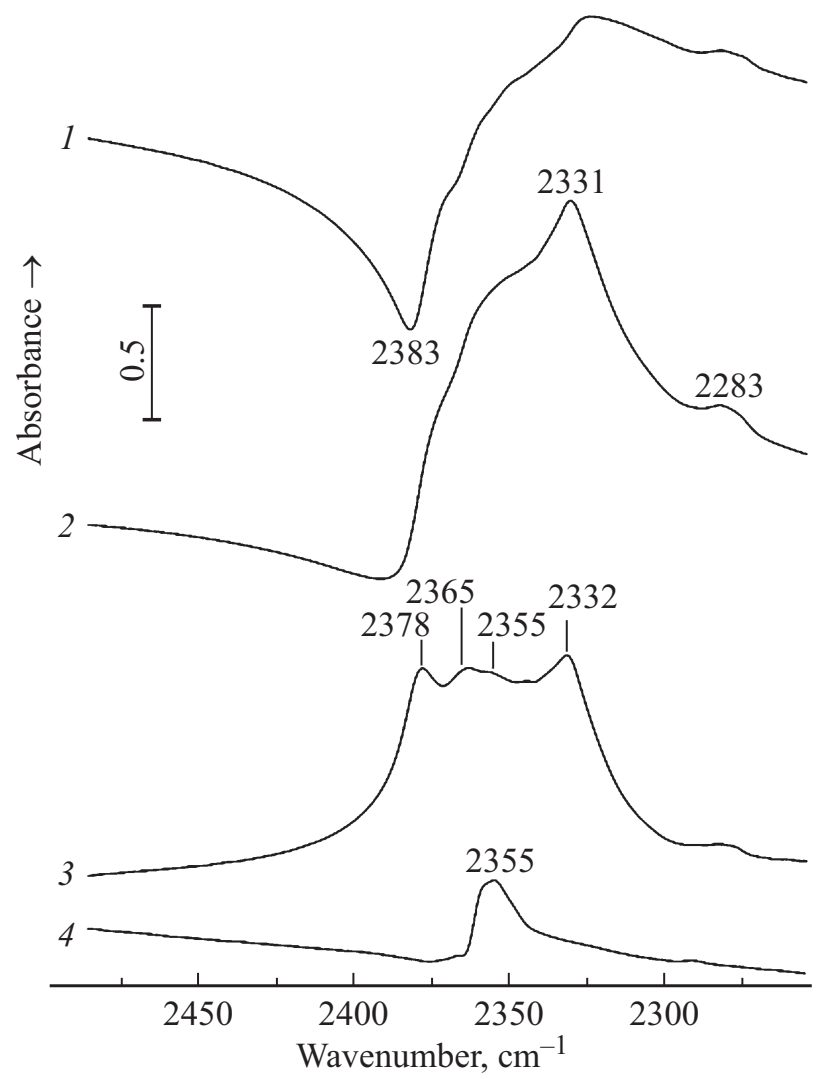

Pис. 4. Спектр поглощения тонкого слоя цеолита $\mathrm{NaX}$, вакуумированного при $673 \mathrm{~K}$, после адсорбции избытка $\mathrm{CO}_{2}$ и охлаждения до 77 К (1); погружения в жидкий кислород (2); результат вычитания спектра 1 из спектра 2 (3); и спектр после десорбции большей части адсорбата при 200 К (4).
Моделирование влияния РДД взаимодействия на спектр показало [2], что его спектральные проявления существенным образом отличаются для одномерной цепи взаимодействующих молекул, слоя плоско расположенных молекул или трехмерной фазы. Таким образом, после учета искажений, вносимых рассеянием, можно исследовать контур полосы поглощения адсорбированных молекул в условиях насыщения поверхности и получать информацию о геометрии поверхности микрочастиц мелкодисперсных образцов.

\section{Выводы}

Анализ полученных данных показывает, что в ИК спектрах поглощения сильно рассеивающих образцов влияние рассеяния не ограничивается ослаблением интенсивности проходящего света, которое в случае изучения спектров адсорбированных молекул можно учесть вычитанием спектра до адсорбции. Вследствие зависимости рассеяния от показателя преломления исследуемого вещества, который изменяется в области полос поглощения, могут происходить существенные искажения контура спектральных полос.

На примере диоксида углерода, адсорбированного на цеолите $\mathrm{NaX}$, продемонстрировано два метода учета вклада рассеяния в спектры поверхностных соединений: регистрация спектра „диффузного пропускания“ и погружение образца в иммерсионную жидкость, в качестве которой использован жидкий кислород.

В спектрах поглощения диоксида углерода, адсорбированного в условиях высокого заполнения поверхности на цеолите $\mathrm{NaX}$, полоса антисимметричного валентного колебания сильно уширена и обнаруживает сложную структуру, возникающую в результате РДД взаимодействия между адсорбированными молекулами. Исследование влияния РДД взаимодействия на спектр диоксида углерода, адсорбированного на других поверхностях, продолжается.

Работа выполнена при финансовой поддержке по гранту РФФИ № 17-03-01372

\section{Список литературы}

[1] Murzin P.D., Novikov R.G., Tsyganenko A.A. // Opt. Spectrosc. 2016. V. 121. N 1. P. 10-17. doi 10.1134/S0030400X1607016X; Мурзин П.Д., Новиков Р.Г., Цыганенко А.А. // Опт. и спектр. 2016. Т. 121. № 1. P. $12-19$.

[2] Dobrotvorskaia A.N., Kolomiitsova T.D., Petrov S.N., Shchepkin D.N., Smirnov K.S, Tsyganenko A.A. // Spectrochim. Acta. A: Mol. Biomol. Spectrosc. 2015. V. 148. P. 271-279. doi 10.1016/j.saa.2015.04.002

[3] Dobrotvorskaia A.N., Gatilova A.V., Murzin P.D., Rudakova A.V., Shchepkin D.N., Tsyganenko A.A. // J. Photochem. Photobiol. A: Chem. 2017 (in press). doi 10.1016/j.jphotochem.2017.09.066 
[4] Martra G. et al. // Res. Chem. Intermed. 1999. V. 25. N 1. P. 77-93. doi 10.1163/156856799X00392

[5] Garrone E. et al. // J. Chem. Phys. 2002. V. 117. N 22. P. 10274-10282. doi 10.1063/1.1519254

[6] Cheung O., Bacsik Z., Liu Q., Mace A., Hedin N. // Appl. Energy. 2013. V. 112. P. 1326-1336. doi 10.1016/j.apenergy.2013.01.017

[7] Nakamoto $K$. Infrared and Raman Spectra of Inorganic and Coordination Compounds. New York: John Wiley Sons, 1986. Накамото К. Инфракрасные спектры и спектры КР неорганических и координационных соединений. М., Мир, 1991.

[8] Sverdlov L.M., Kovner M.A., Krainov E.P. Vibrational Spectra of Polyatomic Molecules. London: Halsted, 1974. Свердлов Л.М., Ковнер М.А., Крайнов Е.П. Колебательные спектры многоатомных молекул. М., Наука. 1970.

[9] Tolstoy V.P., Chernyshova I.V., Skryshevsky V.A. // Handbook of Infrared Spectroscopy of Ultrathin Films. John Wiley \& Sons, Inc., 2003.

[10] Tsyganenko A.A. // Top. Catal. 2013. V. 56. N 11. P. 905-913.

[11] Tsyganenko A.A. // Phys. Chem. Low Temp. Pan Stanford. 2011. N 9. P. 267-296,

[12] Pestsov O.S., Tsyganenko A.A. // Book of Abstracts of 4th International Symposium „Molecular Photonics“ dedicated to academician A.N. Terenin. St. Petersburg, 2016. P. 44. 